\title{
Nature of contact deformation of TiN films on steel
}

\author{
S. Bhowmick \\ Department of Metallurgy, Indian Institute of Science, Bangalore 560012, India \\ Z-H. Xie and M. Hoffman \\ School of Materials Science and Engineering, The University of New South Wales, \\ Sydney NSW 2052, Australia \\ V. Jayaram ${ }^{\text {a) }}$ \\ Department of Metallurgy, Indian Institute of Science, Bangalore 560012, India \\ S.K. Biswas \\ Department of Mechanical Engineering, Indian Institute of Science, Bangalore 560012, India
}

(Received 19 October 2003; accepted 18 May 2004)

\begin{abstract}
Nanoindentation experiments were carried out on a columnar $\sim 1.5-\mu \mathrm{m}$-thick TiN film on steel using a conical indenter with a 5- $\mu \mathrm{m}$ tip radius. Microstructural examination of the contact zone indicates that after initial elastic deformation, the deformation mechanism of the TiN is dominated by shear fracture at inter-columnar grain boundaries of the TiN film. A simple model is proposed whereby the applied load is partitioned between a deforming TiN annulus and a central expanding cavity in the steel substrate. It is possible to obtain a good fit to the experimental load-displacement curves with only one adjustable parameter, namely the inter-columnar shear fracture stress of the TiN film. The implication of results in the context of the performance of TiN films in service is also discussed.
\end{abstract}

\section{INTRODUCTION}

Among the many types of nanoindentation experiments that have been carried out on thin films on substrates, the deconvolution of film hardness has posed special problems. Applications have dictated the filmsubstrate combinations as to whether it is soft on hard or hard on soft. "Soft" and "hard" may be further qualified as to whether these are brittle or ductile. Models to describe and analyze thin film performance have therefore tended to be combination specific ${ }^{1-5}$ and may be classified into two generic categories; (i) where plastic flow is emphasized and (ii) where fracture is emphasized. The focus of our attention is a combination of a hard and brittle film on a ductile substrate; a common example of which are TiN coatings on steel where one typically encounters films of 1-5 $\mu \mathrm{m}$ thickness of a columnar TiN with strong texture, often with the $\langle 111\rangle$ crystal plane normal to the surface. In such cases, it is routine to extract elastic modulus by fitting depth-sensing indentation curves with low maximum loads. However, significant plastic deformation of the hard coating, particularly in

\footnotetext{
a) Address all correspondence to this author. e-mail: qjayaram@met.iisc.ernet.in DOI: $10.1557 / J M R .2004 .0339$
}

thin $(1 \mu \mathrm{m})$ coatings, is very likely preceded by irreversible deformation of the substrate even at a penetration depth (normalized by the coating thickness) of $0.05 .^{6}$ The premise of the present work is that, to the best of our knowledge, there has been no attempt to link microstructural observations which reveal the nature of deformation beneath an indentation to a model that satisfactorily reproduces, not just the final contact size after unloading, but the full load-displacement response of the filmsubstrate combination in TiN films on a ductile substrate.

A variety of models have been applied to elucidate this particular problem, i.e., how does one extract a material property of TiN, the hardness, from load-displacement curves in which the dominant displacements arise out of plastic flow in the substrate, even at an early stage of the loading. One class of models uses a rule of mixtures which assigns a plastically deforming volume to each phase and obtains the net hardness by a linear average. ${ }^{1,2}$ This kind of approach, generally applied to sharp indenters, dates back to the determination of hardness in cermets in which models were developed based on iso-stress or iso-strain approximations from which the composite yield stress was scaled with the volume fraction of each phase. The earliest models to be applied to thin film indentation, which predate the use of depth-sensing nanoindentation, are proposed by Buckel, ${ }^{7}$ Burnett and Page, ${ }^{8}$ 
Sargent, ${ }^{9}$ Jonsson and Hogmark, ${ }^{1}$ and Burnett and Rickerby. ${ }^{2}$

In parallel, analyses of contact fracture in thin films invariably assume that the TiN is predominantly elastic. For example, it has been argued that it behaves as a membrane that conforms to the plastic deformation of the substrate leading to cracks that originate from the interface and at the edge of the contact. Through-thickness cracking of columns at the edge of the contact has been assumed for $\mathrm{TiN}^{10}$ and for TiAlN films ${ }^{11}$ on ductile substrates. Such cracking due to sliding of the columns immediately below the contact was shown for TiN films on a brittle Si substrate. ${ }^{12,13}$ Discontinuities in the loaddisplacement response have been linked to specific fracture events ${ }^{11,14,15}$ and flow or deformation theory in conjunction with the finite element method has been used to determine strains as a prelude to predicting the onset or propagation of cracks. ${ }^{11,16-19}$

The present paper explores spherical nanoindentation on TiN/steel followed by detailed microscopy of the contact zone. This allows us to identify the principal dissipative process in the TiN film. We use this knowledge to build a simple analytical model that seeks to quantitatively describe the load-displacement behavior. The hardness that is extracted from experimental data appears to have little or nothing to do with yielding in TiN, in the conventionally understood manner of dislocation slip at a critical resolved shear stress, as would be expected for a bulk ceramic. We also indicate that the response of a thicker film is qualitatively different and cannot be extrapolated from the behavior seen in thin films.

\section{EXPERIMENTAL PROCEDURE}

Cathodic arc evaporation with titanium targets was used to deposit TiN films on 304 stainless steel substrates whose hardness was determined by conventional microindentation to be $\sim 3 \pm 0.15 \mathrm{GPa}$. The substrates were first polished to an average surface roughness $\left(R_{\mathrm{a}}\right)$ of $\sim 20 \mathrm{~nm}$ and then cleaned thoroughly with solvent and dried before being placed on a continuously rotating planetary holder inside the vacuum chamber. The substrates were heated using radiant heating to the deposition temperature of $350{ }^{\circ} \mathrm{C}$. After the chamber was evacuated to a pressure of $1.3 \times 10^{-3} \mathrm{~Pa}\left(10^{-5}\right.$ Torr $)$, the substrates were sputter cleaned with $\mathrm{Ar}^{+}$and coated with a thin layer of Ti $(\sim 50 \mathrm{~nm})$ to improve the adhesion of the TiN film. Deposition of TiN was carried out in high purity nitrogen at a pressure of $2.6 \mathrm{~Pa}$ (20 mTorr). A negative bias voltage of $150 \mathrm{~V}$ was applied to the substrates during deposition. A film thickness of $1.5 \mu \mathrm{m}$ was achieved after 45 min of deposition. The as-deposited structure of the film contains columns (Fig. 1) of $\sim 0.2 \mu \mathrm{m}$ diameter. X-ray diffraction (XRD) (not shown) reveals a complete $\langle 111\rangle$ texture along the growth direction. ${ }^{20}$

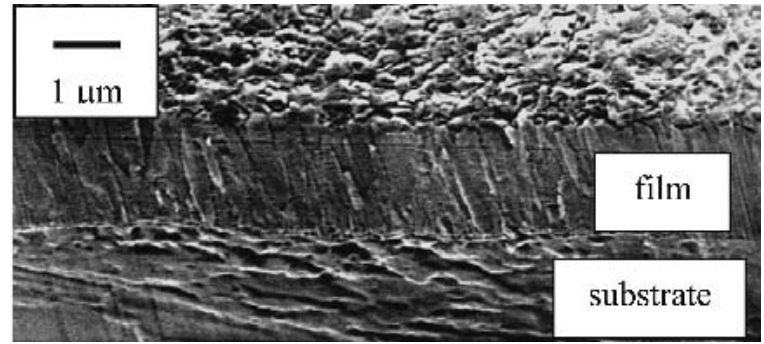

FIG. 1. Secondary electron FIB image of TiN film on steel substrate cross section showing columnar grain structure $(\sim 0.2 \mu \mathrm{m})$.

Cross-sections of the film and substrate were prepared and imaged using a focused ion beam (FIB) mill for characterization of the microstructure and deformation following nanoindentation. This technique is described in detail elsewhere. ${ }^{21}$ The indented surface was examined by both conventional and field-emission scanning electron microscopy (FESEM). X-ray diffraction was used to establish texture. Instrumented nanoindentations were carried out with the Ultra-Micro Indentation System (UMIS - CSIRO Sydney, Australia). A conical diamond indenter with a nominal tip radius of $5 \mu \mathrm{m}$ was used with maximum loads ranging from 10 to $500 \mathrm{mN}$. Loading and unloading were each undertaken over 50 steps with each step taking $0.1 \mathrm{~s}$ followed by a $0.1 \mathrm{~s}$ hold. Resolution of the nanoindenter is limited by thermal drift which was found to be less than $2 \mathrm{~nm} / \mathrm{min}$.

\section{RESULTS}

\section{A. Nanoindentations}

Typical nanoindentation load-displacement $(P-h)$ curves are shown in Figs. 2(a) and 2(b). They are characterized by the following features:

(i) At low loads $(10 \mathrm{mN})$, there is a steep rise in load with displacement from the beginning of loading characterized by an exponent $n\left(P \propto h^{n}\right)$ of 1.5 , indicating essentially elastic Hertzian contact.

(ii) At higher loads $(>50 \mathrm{mN})$, the $P$ - $h$ curves are repeatable in both loading and unloading as shown in Fig. 2(b). Here, with increasing $h$, the exponent $n$ once again drops from a value of 1.5 to about 1 because of other irreversible deformation which is discussed later.

At low loads $(<50 \mathrm{mN})$, the completely elastic nature of the contact is evident in the absence of any permanent impression. At higher loads, the predominant feature of the deformation in the impression is the slippage of columns by shear so as to allow the film to conform to the shape of the indenter. These steps may be clearly seen in cross-sections as shown in Fig. 3(a) especially at the film-substrate interface. On the free surface of the impression this shear deformation reveals itself as concentric rings [Fig. 3(b)].

Significantly, evidence of plastic deformation of the 

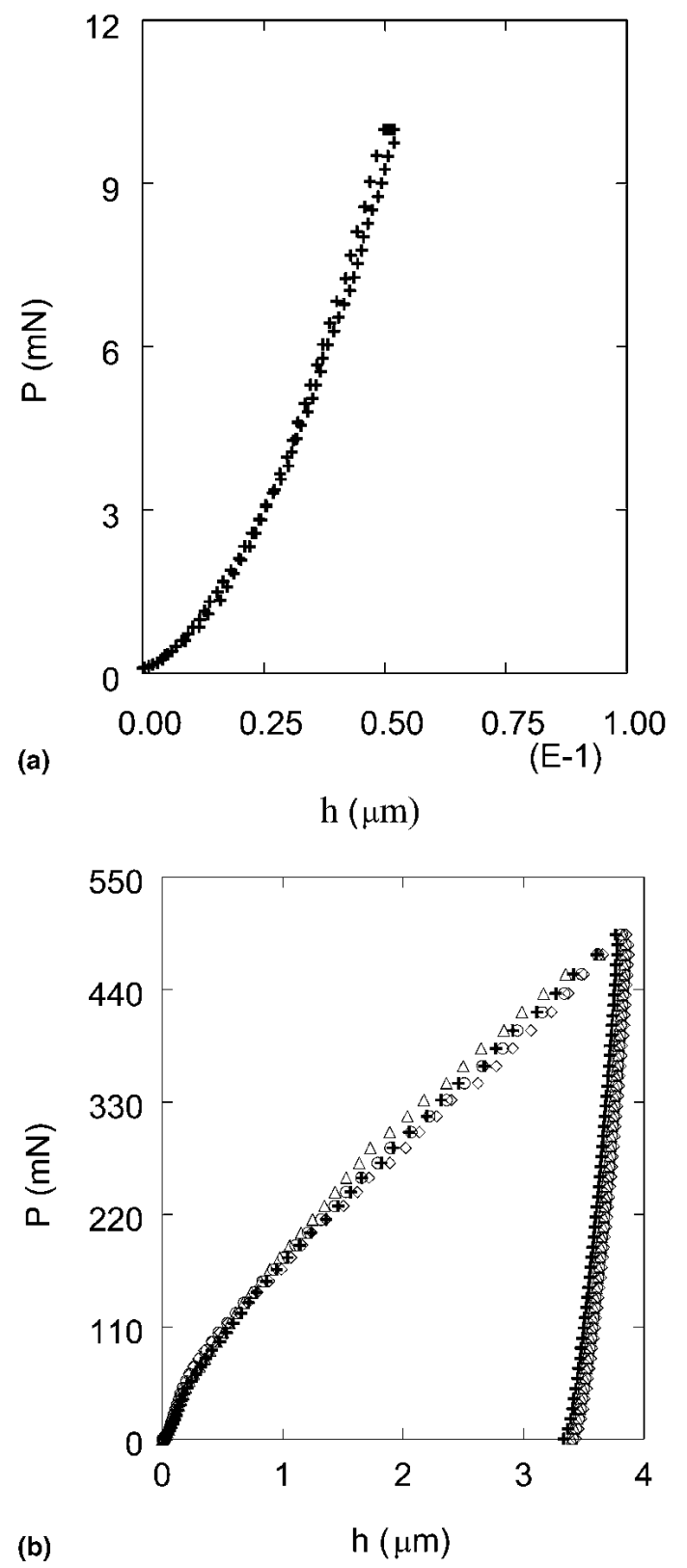

FIG. 2. Typical nanoindentation curves at (a) low load $(10 \mathrm{mN})$ and (b) high load $(500 \mathrm{mN})$.

film is absent. There is no pileup and no measurable thinning of the columnar grains under the indentation. Additionally, transmission electron microscopy images of such a deformed film have shown no difference in the structure and volume of dislocations in the film under the indent or in other regions. ${ }^{22}$ No slip bands which would disturb the planarity of the inter-column boundaries have been observed.

\section{B. Analysis}

The primary thrust of our analysis is the portion of the load-displacement curve beyond the elastic part of the

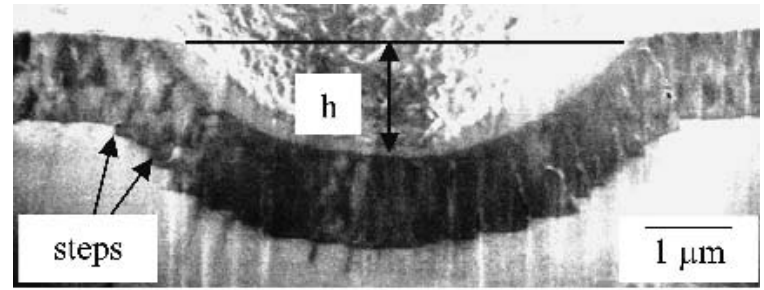

(a)

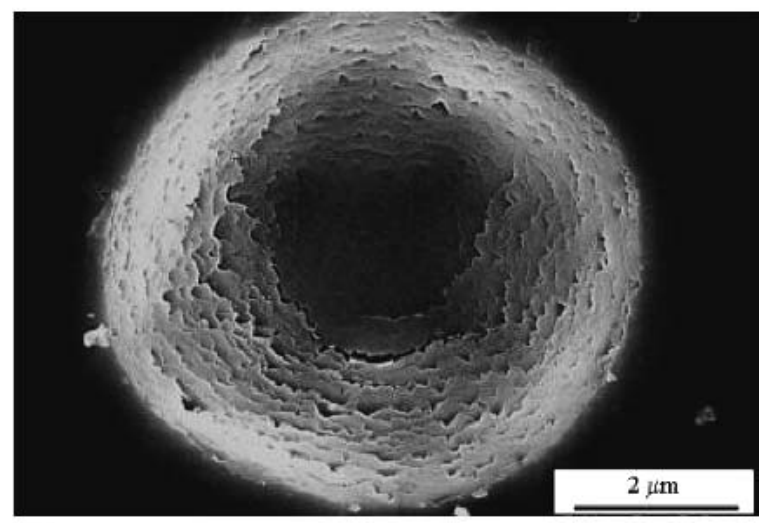

(b)

FIG. 3. (a) FIB image of cross section showing shear slipping of columns with column spacing $\sim 0.2 \mu \mathrm{m}$ and (b) SEM image of an impression showing concentric rings of cracks on the free surface at $500 \mathrm{mN}$ load. The cross section image was captured at a tilt angle of $45^{\circ}$.

loading. Given the microstructural evidence, in particular, the obvious appearance of slip steps at the intercolumn boundaries, the absence of significant plastic thinning of the film and the lack of experimental evidence for gross plastic flow across TiN columns, it is proposed here that the overall deformation is accompanied by a process of inter-columnar shear cracking of the TiN, which makes the central caved-in part of the film an extension of the spherical indenter (Fig. 4).

It may be noted that the inter-columnar interfaces do not display significant opening displacements normal to the column direction. The present observation suggests the inference that the slipping of columns is related to a sliding action and not to tensile stresses. The proposition that the film is load bearing only at the edge of the column was made in models that sought to fit rules of mixtures to the composite hardness. ${ }^{1,2}$ Similarly, Whitehead and Page ${ }^{5}$ have, in their analysis of fracture, assumed that the film deforms elastically and that the coating acts as a cap on the indenter once the "nested" crack at the edge of the contact propagates around the circumference and separates the inside portion from the rest of the film.

The novelty in our approach is to use these observations to model the load-displacement curve as a combination of: (i) the load required to bring permanent shear displacement in the film at the edge of contact and (ii) the 


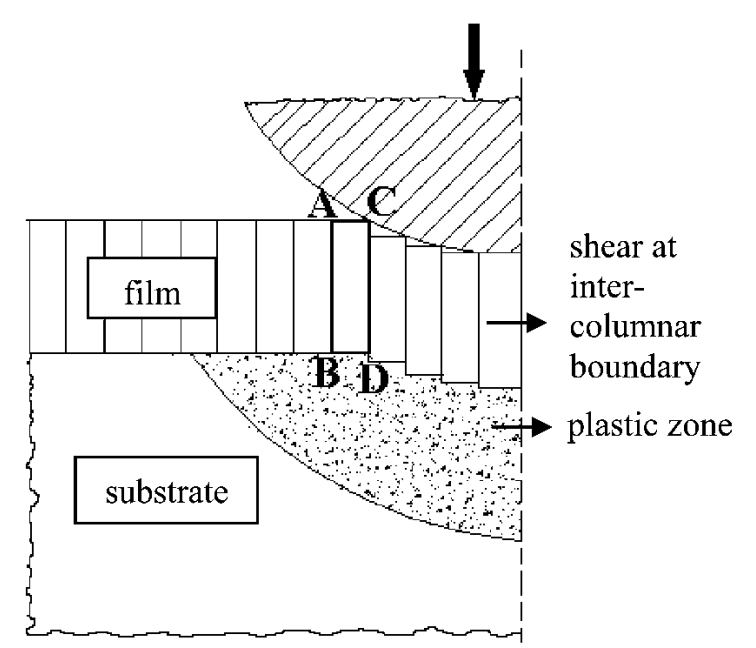

FIG. 4. Schematic of the deformation of the film-substrate system. AB is the active inter-columnar boundary supporting a part of the total load, whereas the remaining load transfers to the substrate through the inter-columnar boundaries (including the boundary $\mathrm{CD}$ ), which have undergone shear fracture.

load required to indent the substrate by the extended indenter. The critical event that relates the film and the substrate is assumed to be the shearing of the columns at the contact edge, at a critical stress, which is the only unknown parameter in the analysis. As the load increases, the column at the outer edge cracks when the shear strain reaches a critical value, thereby allowing the indenter to further penetrate the substrate and, at the same time, make contact with the next ring of columns which had hitherto been outside the deformed zone.

\section{Loading}

We first model the low load behavior. Figure 5 shows that when the indenter first contacts with TiN, i.e., $2 a \ll d$. The experimental $P$ - $h$ curve in this load regime $(0-$ $23 \mathrm{mN}$ ) is accurately estimated by assuming a purely elastic Hertzian contact. This implies that the initial contact area covering about 5-6 columns is elastic. From several experiments conducted up to a load of $10 \mathrm{mN}$ the Young's modulus is estimated to lie in the range 350$450 \mathrm{GPa}$ which compares well with the value of $400 \mathrm{GPa}$ found earlier, ${ }^{23}$ and the scatter is expected since the initial contact may take place in the vicinity of an intercolumnar boundary.

Beyond the very initial stage $(2 a \ll d)$ of the indentation process, the film can no longer be regarded as a semi-infinite half space, thereby ruling out a Hertzian description of the elastic response of the film. We model the overall deformation as consisting of two uncoupled parts: (i) elastic response of the film of finite thickness resting on a rigid substrate and (ii) the elasto-plastic response of a film/substrate where the film response is

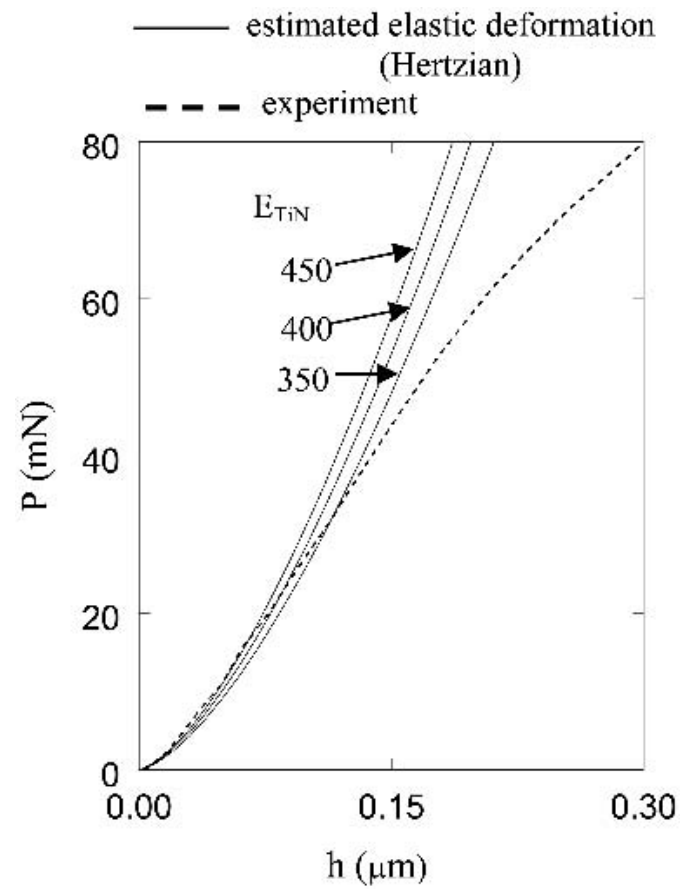

FIG. 5. Elastic portion of TiN curve (experiment) with predicted model.

purely through the plastic shear of inter-columnar boundaries at the edge of contact, and where the substrate is being indented elasto-plastically. To determine the global response we bring the two parts together by coupling a set of springs of known stiffness.

\section{a. Elastic response of the film}

We consider the film as a slab of finite area of finite thickness resting on a rigid frictionless foundation and compressed by a spherical indenter. We use the numerical analysis of Conway and Engel ${ }^{24}$ to arrive at a stiffness of the film of elastic modulus $\sim 400 \mathrm{GPa}$, being indented by a spherical indenter of radius $5 \mu \mathrm{m}$.

$S_{\mathrm{e}}=\mathrm{d} P / \mathrm{d} h_{\mathrm{e}}=1393.5+10367.4 h_{\mathrm{e}}-2434 h_{\mathrm{e}}{ }^{2}$,

where $h_{\mathrm{e}}$ is the indenter displacement in a direction coaxial to load application, $h_{\mathrm{e}}$ is in $\mu \mathrm{m}$, and $S_{\mathrm{e}}$ is in $\mathrm{mN} / \mu \mathrm{m}$. We recover the Hertzian stiffness from Eq. (1) only when $h_{\mathrm{e}}<0.03 \mu \mathrm{m}$.

\section{b. Elasto-plastic response of the film/substrate}

Since the displacements of the film and the substrate, when the deformation of the film is highly localized at the edge and the film does not undergo any permanent thinning, are always about the same, it is assumed that the indentation load is shared between the two elements. In essence, in response to an incremental displacement of the indenter, the central portion of the columns pushes into the substrate, but only to the extent determined by 
the permanent strain at the interface between the columns inside and those just outside the contact. Clearly, the surface of the steel cavity is displaced correspondingly. (At the other extreme, when both the film and substrate deform globally, as for example in the case of a soft film on a hard substrate, the most logical coupling is that between two serial springs displacing differently while being subjected to the same load. In more general systems, where both localized and global deformations are involved, the coupling may be a combination of these two extreme configurations). While there are similarities to this additive concept in earlier models of hardness, the present work perhaps represents the first attempt to apply the model in the manner elaborated below. Thus, the film at the contact edge supports a load $\mathrm{P}_{1}$ and the substrate supports an indentation load $P_{2}$ such that the applied load $P_{\mathrm{h}}=P_{1}+P_{2}$. We estimate $P_{1}$ and $P_{2}$ as follows:

(i) The load required to fracture an inter-columnar boundary at the edge of the contact of indentation is $P_{1}$. Considering a critical inter-columnar failure stress in shear to be $\tau^{*}$

$$
\mathrm{P}_{1}=2 \pi \mathrm{at} \tau^{*}
$$

$\mathrm{a}$ is a function of $\mathrm{h}$ as follows

$$
a=\sqrt{2 R_{\mathrm{i}} h-h^{2}} .
$$

A spring of stiffness $S_{1}\left(S_{1}=\mathrm{d} P_{1} / \mathrm{d} h\right)$ may be considered to represent the resistance to shear fracture of TiN as shown in Fig. 6.

(ii) The load required to deform the substrate elastoplastically is $P_{2}$. Since the film acts as a strap to the indenter, we take into account the extended indenter and define $P_{2}$ using the expanding cavity model ${ }^{25}$ as

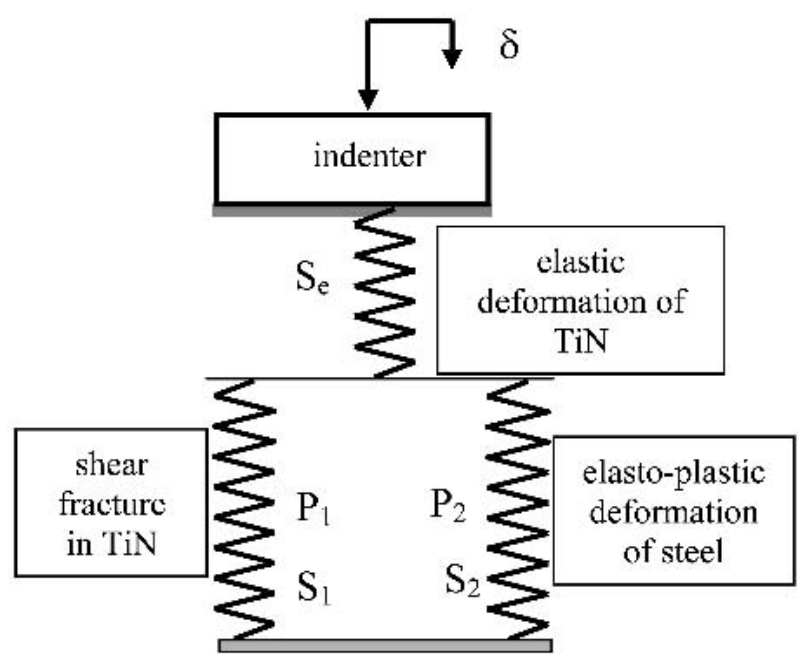

FIG. 6. A combination of springs which is used to model the deformation of the film-substrate system under indentation.

$$
P_{2}=\frac{2 \pi\left(a^{\prime}\right)^{2} Y_{\mathrm{s}}}{3}\left\{1+\ln \left(\frac{1}{3} \frac{E_{\mathrm{s}}^{a^{\prime}} / R_{\mathrm{i}}^{\prime}}{Y_{\mathrm{s}}}\right)\right\} .
$$

The elasto-plastic deformation of the substrate is represented by a spring of stiffness $S_{2}\left(S_{2}=\mathrm{d} P_{2} / \mathrm{d} h\right)$, which acts in parallel with the spring of stiffness $S_{1}$ in Fig. 6 . Thus, the equivalent stiffness of this parallel spring configuration $\left(S_{\mathrm{h}}\right)$ is defined as

$$
\mathrm{S}_{\mathrm{h}}=\mathrm{S}_{1}+\mathrm{S}_{2} \text {. }
$$

We now couple the two deformations to give the overall deformation and global stiffness as

$$
\begin{aligned}
\mathrm{h} & =\mathrm{h}_{\mathrm{e}}+\mathrm{h}_{\mathrm{h}}, \\
\frac{\mathrm{d} P}{\mathrm{~d} h} & =S_{\mathrm{eq}}=\left(\frac{1}{S_{\mathrm{e}}}+\frac{1}{S_{\mathrm{h}}}\right)^{-1} .
\end{aligned}
$$

By substituting $S_{\mathrm{e}}$ and $S_{\mathrm{h}}$ from Eqs. (1) and (5) in the above equation, we estimate the entire loaddisplacement numerically. It may be worthwhile to point out at this stage that the elastic deformation of the film $h_{\mathrm{e}}$ is indeed an insignificant part of the overall deformation $h$ at high loads (e.g., $300-500 \mathrm{mN}$ ), but is significant in the low load region where it approaches the Hertzian prediction. ${ }^{24}$ We thus keep this elastic spring as an integral part of our model to describe the global response of the film/substrate system over a wide range of indentation load. Taking the elastic modulus of the TiN column to be $400 \mathrm{GPa}$ and the modulus and yield stress of the steel substrate to be 210 and $1 \mathrm{GPa}$, respectively, we fit an estimated $P$ - $h$ (loading) characteristic to the experimental result (Note: independent measurements of the load-displacement curves on the bare substrate show that the expanding cavity model fits well with these values). Figure 7(a) shows that the model fits well the experimental curves for a range of $\tau^{*}$ of 2.3-3.1 GPa. By fitting the repeated experimental curves, an average $\tau^{*}$ is found to be $2.8 \mathrm{GPa}$. It is important to note that the fit between experiment and model is possible (i) without invoking any mechanism of conventional dislocation assisted yielding of TiN and (ii) by sharing out the load (additively) between the inter-columnar shear at the contact edge and the elastic-plastic deformation of the substrate, in proportion to their respective mechanical properties.

\section{Unloading}

We model the unloading behavior of the film-substrate system at high loads $(2 a \gg d)$ assuming that displacements of the substrate and the film are both purely elastic and additive. Accordingly the overall displacement during unloading is given by

$$
\Delta \mathrm{h}=\delta_{1}+\delta_{2},
$$

where $\delta_{1}$ is the unloading vertical displacement at the central region of the spherical (TiN) cup of inner radius $R_{\mathrm{i}}$ and thickness $t$. 
Assuming the cup is a segment of a thick spherical shell, we may approximately write ${ }^{26}$

$$
\delta_{1}=\frac{\frac{P}{E_{\mathrm{f}}}\left[(1-2 v)+\frac{(1+v)\left(R_{\mathrm{i}}+t\right)^{3}}{2 R_{\mathrm{i}}^{2}}\right]}{\left[\left(\frac{R_{\mathrm{i}}+t}{R_{\mathrm{i}}}\right)^{3}-1\right]},
$$

where $\delta_{2}$ is the unloading vertical displacement at the central region of the spherical (steel) cavity indented into the substrate; $\delta_{2}$ may again be estimated approximately using the above equation by considering $t=\infty$. Figure 7(b) shows that the estimated unloading characteristics follow the experimental characteristics closely for maximum loads of 100,200 , and $500 \mathrm{mN}$. We may note that the recovery slope increases with load and this is well indicated by the assumed model.

In summary, beyond the elastic limit, a TiN strap attaches itself to the indenter and the effective radius of the indenter increases by the TiN film thickness. During the course of indentation, as the film enters the zone under the indenter it undergoes large strain. The inter-columnar interface takes the strain as the weakest link and there is shear fracture such that the entering TiN column can conform to the indenter shape to become the "strap" for subsequent penetration, thus leading to the creation of steps at the interface with the substrate and at the free surface. Thus, the critical and most important response of TiN comes from the inter-columnar spacing and the inter-columnar failure stress.

It is also important to address the issues of the status of the TiN strap under the indenter. No permanent strain takes place in the columns that have already cracked. They are compressed elastically as cylinders. The equilibrium normal pressure on a column at the center of the film is the spherical cavity (steel) pressure which declines as one moves away from the center. As the columns are compressed between the steel and the indenter there will be bending stresses in the film tangential to the indenter profile. These stresses have been shown to be tensile at the steel-TiN interface and compressive at the top surface. ${ }^{11}$ These stresses are also maximum at the central indentation axis. Tensile failure of the intercolumnar interface will thus start at the TiN-steel interface and propagate toward the indenter without penetrating the compressive zone near the surface. We do not see these cracks at low loads but do see them at high loads. ${ }^{22}$

\section{DISCUSSION}

Our results have significant implications for the evaluation of hardness of films on substrates. The hardness of a bulk solid is a measure of resistance to dissipative
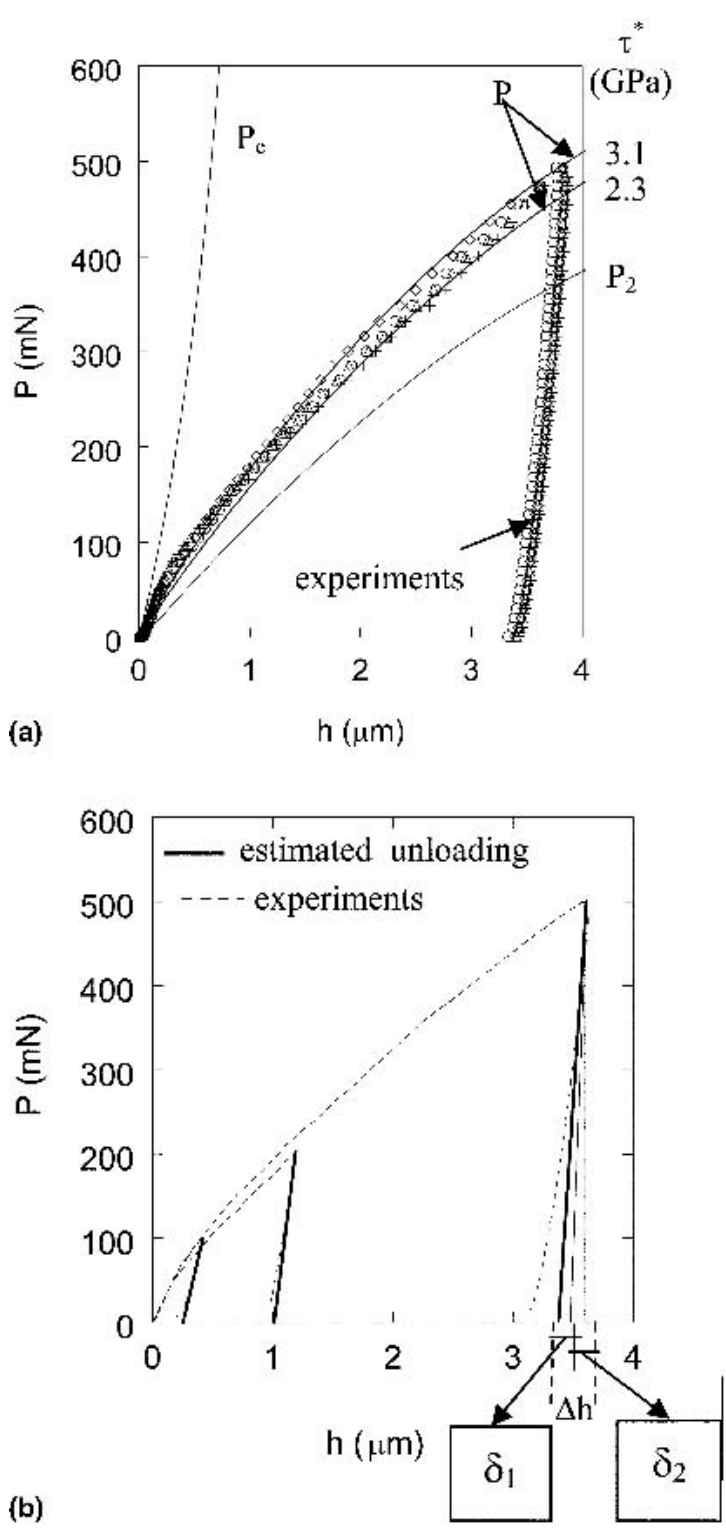

FIG. 7. (a) Full elastic-plastic curve with model predictions for various inter-columnar strengths $\tau^{*}$ and (b) the elastic recovery of the film and substrate.

processes such as dislocation glide and/or fracture. For a film-substrate system, the peak load and permanent deformation, the two measured parameters used for hardness estimation are clearly composite responses to the principal dissipative process in the film and in the substrate. In the present case, a measured hardness is therefore a composite of a critical stress to fracture a grain boundary (albeit a preferential one, given the strong $\langle 111\rangle$ texture) in the film and the stress associated with dislocation glide in the substrate. A hardness value estimated in the conventional way using peak load and permanent deformation does not therefore yield any useful information unless the recorded data is deconvoluted using realistic models of dissipation to yield highly structure 
specific information such as inter-columnar shear strength.

In extending our model to thicker films, it is clear that when the interface is remote from the contact that it is unlikely that the shear cracks at the edge of the contact will propagate all the way down the column. One can roughly estimate the depth of such a crack by balancing the driving force with the toughness using expressions for an infinite crack in a solid. Consider a circular crack of radius $a$ and length $L$ that is subjected to a shear (mode II) displacement $u$, at the root that is given by the shear strain at the edge of the spherical contact. We may assume that $u$ is the difference in displacement of the column at the edge of the contact (i.e., at $a$ ) when the first shear crack appears, with respect to that of the previous column. If the depth at which shear cracking begins is $\sim 0.1 \mu \mathrm{m}$ (this corresponds to the onset of a change in the exponent of the load-displacement curve), then $u \sim 0.06 \mu \mathrm{m}$ for an indenter tip radius of $5 \mu \mathrm{m}$. The driving force available for crack extension is given by

$$
G=K^{2} / E, \text { where } \mathrm{u} \sim 2 K(L)^{1 / 2} / \mu,
$$

Thus, $G \sim E u^{2} / 16 L$.

The crack will extend until it runs out of driving force, i.e., $G \sim G_{\mathrm{c}}$, whereupon $L=E u^{2} / 16 G_{\mathrm{c}}$. Inserting $E=400 \mathrm{GPa}$, one obtains $L \sim 8 \times 10^{-5} / G_{\mathrm{c}}$, which yields for $G_{\mathrm{c}} \sim 100 \mathrm{Jm}^{-2}$ (corresponding to $K_{\mathrm{c}} \sim 6 \mathrm{MPa} \mathrm{m} \mathrm{m}^{0.5}$ ) a crack length of $1 \mu \mathrm{m}$; i.e., the entire length of the column will be cracked. Thus, in thicker films, shear cracking along the column may well be confined to the near surface region, and the part of the column below can continue to support an increasing load, which can then lead to a different mode of fracture.

While we have not explicitly addressed cracking in the present work (other than the shear that leads to intercolumnar slip), it is worthwhile to point out that thin and thick films can display different damage behavior. In this context, the observation made in our present work may be compared to the damage discussed in our previous paper $^{27}$ in the case of a $5 \mu \mathrm{m}$ TiN film deposited on stainless steel and to the damage observed by Lee et al. ${ }^{28}$ in much thicker coatings of $250 \mu \mathrm{m}$ in an isotropic ceramic film. The micrographs in Figs. 3 and 8 compare the effect of coating thickness on the damage pattern for a similar structure of columnar TiN coatings. In the thicker film, the edge cracks do not penetrate deep into the film (Fig. 8 and sequential polishing experiments described elsewhere in Bhowmick et al. ${ }^{27}$ ), and the inter-columnar shear that is characteristic of our present work does not extend all the way down to the interface. Instead, we observe that a new form of damage marked as "inclined crack" (Fig. 8) originates in the sub-surface of the coating and runs across the TiN columns. Lee et al. ${ }^{28,29}$ also

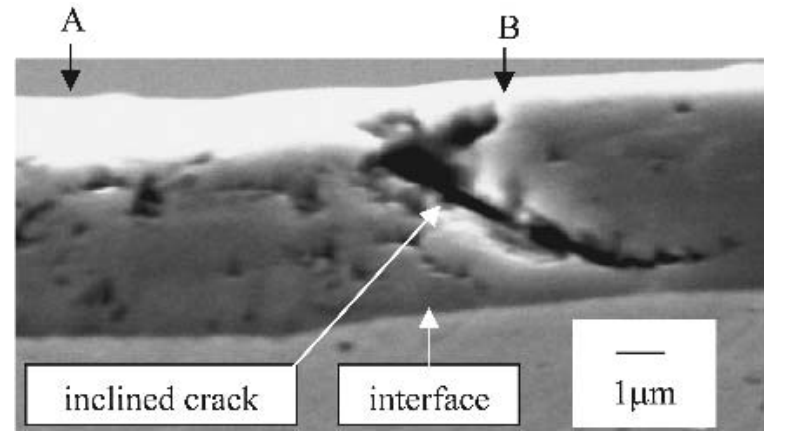

FIG. 8. Cross section of Vickers indentation (A and B indicate center and edge of the impression) in $5 \mu \mathrm{m}$ film showing inclined cracks and a smooth (not stepped) interface.

reported a similar damage which was referred to as an "intermediate crack" and nucleates inside the coating and propagates in both directions, i.e., toward the free surface and also towards the interface.

We believe that the strained volume during indentation loading should be considered an important parameter when the film thickness is a variable. This volume can be considered to scale with the contact radius. Whether a complete shear fracture will occur throughout the film thickness or not, therefore, strongly depends on the ratio of alt. The contact loading in a thick film $(5 \mu \mathrm{m})$ using a Vickers indenter can be considered as a case of small $a / t$. In this case, it is observed (Bhowmick et al. ${ }^{27}$ ) that the inter-columnar shear appears just near the surface and does not extend much into the film. In thinner coatings and larger indenter radii (i.e., large $a / t$ ), the significant amount of plastic flow in the substrate allows the hard coating to act as a strip that follows the indenter geometry, whereas in thicker coatings and smaller indentation radii (i.e., small a/t), for an application of a similar load, the amount of plastic flow in the substrate is reduced, thereby leading to a different distribution of stresses that lead to other forms of cracking. ${ }^{27}$ Thus, the damage in the system does not behave self-similarly with respect to film thickness. Additional important variables include the inter-columnar strength and the yield stress of the substrate, relative to that of the film. While the first may be modified by processing changes, the later is fixed by the application. Both will dictate the thickness at which a film changes from "thin" to "thick": harder substrates and stronger columns will tend to reduce inter-columnar shear and encourage other modes of cracking in the coating; i.e., the film will effectively behave in a "thick" manner.

\section{CONCLUSION}

We now highlight the conclusions and implications of the present work. For columnar TiN films on a plastic substrate, such as steel, detailed microscopy has revealed 
that during nanoindentation, the columnar film structure does not undergo any plastic deformation, but rather, inter-columnar sliding to conform to the shape of the indenter. The indentation load-displacement response is simulated by assuming that this sliding occurs at a critical shear stress so that the central, fully cracked portion becomes a physical extension of the indenter. The applied load is now borne partially by the uncracked cylindrical annulus of TiN and partially by the substrate whose deformation may be modeled as an expanding cavity. A critical inter-columnar shear strength of $2.8 \mathrm{GPa}$ reproduces closely the observed loading curves, while elastic recovery is also accurately predicted by approximating the deformed zone to a segment of a thick spherical shell. No other adjustable parameters are required in this analysis of composite load-displacement behavior.

From a more general standpoint, the major roles of a structural coating are to protect an engineering substrate from environmental corrosion. Substrate corrosion is prevented if the coating, on loading, retains its structural integrity; i.e., loading does not promote interfacial failure and through-thickness fracture. The contact damage of the substrate, on the other hand, is minimized if the coating supports a substantial part of the applied load without failing. We have demonstrated here that thin titanium nitride coatings deposited on steel by physical vapor deposition, which are known to be structurally stable in contact loading, derive their protective role by supporting a part of the contact load in proportion to their intercolumnar failure strength. Our previous study (Bhowmick et al. ${ }^{27}$ ) has shown that as the film becomes thicker, trans-columnar modes of deformation make additional contributions to load support. However, that mode of damage may deteriorate the structural integrity and the stability of the coating. Given that the problem of filmsubstrate interfacial failure has been solved for this coating system by introducing a Ti-based interlayer at the interface, it would appear to be the case that at least for genuinely thin films, the process optimization for more severe applications should take the form of increasing film thickness and strength of the inter-columnar interfaces while maintaining the same mode of shear cracking deformation. Furthermore, if the load support mechanism elucidated here does have a more general validity, it may be possible to design multilayer systems that will substantially diminish substrate damage even further.

\section{ACKNOWLEDGMENTS}

The samples used in this present work have been prepared by Multi Arc (India) Limited, with whom the authors from Indian Institute of Science, have a long standing collaboration. The authors thank Dr. Julie Cairney for assistance with some of the FIB images.

\section{LIST OF SYMBOLS}

$a$ projected radius of contact at the film surface under indentation, $a=\sqrt{\left(2 R h-h^{2}\right)}$

$a^{\prime}$ projected radius of contact at the film/substrate interface under the extended indenter, $a^{\prime}=\sqrt{\left(2 R^{\prime} h-h^{2}\right)}$

$d$ column diameter

E elastic modulus; subscripts i, f, and s represent indenter, film and substrate respectively

$G \quad$ strain energy release rate

$G_{\mathrm{c}} \quad$ critical strain energy release rate

$h$ indentation depth

$K \quad$ stress intensity factor

$K_{\mathrm{c}} \quad$ critical stress intensity factor

$L \quad$ length of the shear crack inside the film

$\mu \quad$ shear modulus of the film

$v$ Poisson's ratio

$P \quad$ indentation load

$R_{\mathrm{i}} \quad$ radius of indenter

$R \mathrm{i} \quad$ radius of extended indenter, $\mathrm{R}+\mathrm{t}$

$S_{\mathrm{e}} \quad$ stiffness of the spring which represents elastic deformation of TiN film

$S_{1} \quad$ stiffness of the spring which represents shear fracture of TiN film

$S_{2} \quad$ stiffness of the spring which represents elasto-plastic deformation of steel

$S_{\text {eq }} \quad$ stiffness of the spring configuration which represents overall deformation

$t \quad$ film thickness

$\tau^{*} \quad$ critical inter-columnar failure (shear) stress of TiN film

$u \quad$ shear displacement of the columns at the edge of the contact

$Y_{\mathrm{s}} \quad$ yield stress of the substrate

\section{REFERENCES}

1. B. Jonsson and S. Hogmark: Hardness measurements of thin films. Thin Solid Films 114, 257 (1984).

2. P.J. Burnett and S. Rickerby: The mechanical properties of wearresistant coatings: I: Modelling of hardness behaviour. Thin Solid Films 148, 41 (1987).

3. P.J. Burnett and S. Rickerby: The mechanical properties of wearresistant coatings: II: Experimental studies and interpretation of hardness. Thin Solid Films 148, 51 (1987).

4. R. Saha and W.D. Nix: Effects of the substrate on the determination of thin film mechanical properties by nanoindentation. Acta Mater. 50, 23 (2002).

5. A.J. Whitehead and T.F. Page: Nanoindentation studies of thin film coated systems. Thin Solid Films 220, 227 (1992).

6. X. Chen and J.J. Vlassak: A Finite Element Study on the Nanoindentation of Thin Films, in Fundamentals of Nanoindentation and Nanotribology II, edited by S.P. Baker, R.F. Cook, S.G. Corcoran, and N.R. Moody (Mater. Res. Soc. Symp. Proc. 649, Warrendale, PA, 2001) Q1.3.1.

7. H. Buckle: The Science of Hardness Testing and Its Research Applications, in Science of Hardness Testing and Its Research Applications, edited by J.H. Westbrook and H. Conrad (ASM, Metals Park, OH, 1973), p. 453. 
8. P.J. Burnett and T.F. Page: Surface softening in silicon by ion implantation. J. Mater. Sci. 19, 845 (1984).

9. P.M. Sargent: Use of indentation size effect on microhardness for materials characterization. In Microindentation Techniques in Material Science and Engineering, edited by P. Blau and B.R. Lawn (ASTM Spec. Tech. Publ., Philadelphia, PA, 1984), p. 160.

10. C. Knight, A.J. Whitehead, and T.F. Page: Nanoindentation experiments on some amorphous hydrogenated carbon $(\mathrm{a}-\mathrm{C}: \mathrm{H})$ thin films on silicon. J. Mater. Sci. 27, 3939 (1992).

11. E. Weppelmann and M.V. Swain: Investigation of the stresses and stress intensity factors responsible for fracture of thin protective films during ultra-micro indentation tests with spherical indenters. Thin Solid Films 286, 111 (1996).

12. E. Weppelmann, M. Wittling, M.V. Swain, and D. Munz: Indentation cracking of brittle thin films on brittle substrates. In Fracture Mechanics of Ceramics 12, edited by R.C. Bradt (Plenum Press, NY, 1996).

13. M. Wittling, A. Bendavid, P.J. Martin, and M.V. Swain: Influence of thickness and substrate on the hardness and deformation of TiN films. Thin Solid Films 270, 283 (1995).

14. M.V. Swain and J. Mencik: Mechanical property characterization of thin films using spherical tipped indenters. Thin Solid Films 253, 204 (1994).

15. D.F. Bahr, J.W. Hoehn, N.R. Moody, and W.W. Gerberich: Adhesion and acoustic emission analysis of failures in nitride films with a metal interlayer. Acta Mater. 45, 5163 (1997).

16. M.R. Begley, A.G. Evans, and J.W. Hutchinson: Spherical impression of thin elastic films on elastic-plastic substrates. Inter. Jl. Solid Struc. 36, 2773 (1999).

17. J.S. Wang, Y. Sugimura, A.G. Evans, and W.K. Tredway: The mechanical performance of DLC films on steel substrates. Thin Solid Films 325, 163 (1998).

18. R.M. Souza, G.G.W. Mustoe, and J.J. Moore: Finite-element modeling of the stresses and fracture during the indentation of hard elastic films on elastic-plastic aluminum substrates. Thin Solid Films 355-356, 303 (1999).

19. S. Sriram, R. Narasimhan, and S.K. Biswas: A numerical fracture analysis of indentation into thin hard films on soft substrates. Eng. Frac. Mech. 70, 1323 (2003).

20. T. Matsue, T. Hanabusa, and Y. Ikeuchi: Residual stress and its thermal relaxation in TiN films. Thin Solid Films 281-282, 344 (1996).

21. Z-H. Xie, P. Munroe, R.J. Moon, and M. Hoffman: Characterization of surface contact-induced fracture in ceramics using a focused ion beam miller. Wear 255, 651 (2003).

22. Z-H. Xie, M. Hoffman, P. Munroe, and R.J. Moon: (2004, unpublished).

23. E. Torok, A.J. Perry, L. Chollet, and W.D. Sproul: Young's modulus of TiN, TiC, ZrN and HfN. Thin Solid Films 153, 37 (1987).

24. H.D. Conway and P.A. Engel: Contact stresses in slabs due to round rough indenters. Int. J. Mech. Sci. 11, 709 (1969).

25. K.L. Johnson: Contact Mechanics (Cambridge University Press, Cambridge, U.K., 1985).

26. R. Hill, The Mathematical Theory of Plasticity (Clarendon, Oxford, U.K., 1950).

27. S. Bhowmick, A.N. Kale, V. Jayaram, and S.K. Biswas: Contact damage in TiN coatings on steel. Thin Solid Films 436, 250 (2003).

28. S.L. Lee, S. Wuttiphan, X. Hu, S.K. Lee, and B.R. Lawn: Contactinduced transverse fractures in brittle layers on soft substrates. A study on silicon nitride bilayers. J. Am. Ceram. Soc. 81, 571 (1998).

29. K.S. Lee, S.K. Lee, D.K. Kim, and B.R. Lawn: Contact damage and strength degradation in brittle/quasi-plastic silicon nitride bilayers. J. Am. Ceram. Soc. 81, 2394 (1998). 